\title{
Selfish Search on Playing Shogi
}

\author{
Takeshi Ito \\ Department of Computer Science, \\ University of Electro-Communications, Tokyo, Japan \\ ito@cs.uec.ac.jp
}

\section{Introduction}

If a human faces a complex problem with a large number of potential solutions and calculations, he will not always act logically. In gamble, public lottery, etc., we see a mechanism by which a bookmaker makes a profit probable. There the chances of winning are always overestimated by the human player. For example, in public lottery, some people are allured by ungrounded rumors (such as "it is the turn of the sales store to win"). Other people take superstitious actions grounded on fortunetelling. A fully different example of irrational human decision making in games can be found in the domain of the imperfect information and the chance gambling game Mahjong. A recent study [1] evaluating game records of internet Mahjong concluded that some amateur players treat a professional's remark like a proverb and believe it blindly in spite of a lack of evidence to support the proposition.

Not only in games of chance, but also in combinatorial game like Chess, Shogi (Japanese Chess) or Go, a human decision is mostly not necessarily founded on rational thinking. Many players are tending to select a candidate move intuitively based on their knowledge and experience. The study of Chess by De Groot is a famous example [2]. In previous work, we conducted mental experiments in the domain of Shogi and replicated De Groot's research on the human intuitive thinking process in the domain of Shogi $[3,4,5,6]$.

Computer programs perform the decision-making tasks in combinatorial games such as Shogi in a quite different from human players. Here we have two opposing systems that deserve further research.

First, human experience cannot be treated as intuitive knowledge. So, we have to address the human search process in another way than by heuristics only. Second, the computer is arriving at a (best) move by the virtue that its search process is based on a rigidly structured search. In computer Shogi, the technique of finding the best move happens by the alpha-beta game-tree search. It is based on an evaluation function that is widely used. The approach is known as "a search driven system" [7].

Computer Shogi avoided incorporating human heuristics as much as possible (since intuition is considered to be more important than experience), and has developed the technology for searching deeply, fast and wide [8]. In the last decade we see that Shogi programs are becoming stronger with the progress of the hardware technology. The playing strength of the top class Shogi programs is said to roughly equal the strength of 6-dan amateur players. It reaches the level of professional players [9].

However, strong Shogi programs play sometimes moves which are perceived of as unnatural by human players. As mentioned above, a significant difference exists 
between the decision-making process of humans and computers. A reason is that computer programs, in spite of playing strongly, suffer from intuition. Their style of play may be uninteresting and not useful to study for a human player. This might be so because a player does not understand the meaning of the move played by the computer. Some programs are able to display a part of their search log to show the 'meaning' of a particular move. However, since the move decision process is not presented completely, it may remain unintelligible as a whole, and the human player cannot understand it.

In order to show a decision-making process in a form understandable to a human, it is necessary to clarify the thought process that human players are performing. In this article, we show what kind of search process is carried out in the human thought process which generates a move when playing Shogi.

Based on interviews with human expert players and verbal protocol data of human players of various levels, we will discuss in particular the "mechanism of prediction" peculiar to human players.

\section{Some Results from Psychological Experiments}

\section{(1) Reducing the set of candidate moves to a few moves}

Figure 1 expresses the average number of moves mentioned as candidate moves at the time of determining the next move and making the human being to think freely on the next move [6]. In the figure we see that the amateur 1-DAN (middle level players) raised many candidate moves. In average they still arrived at five whereas there are only three candidate moves. Since the problem given here is the position where a legal move exceeds 100 hands from tens hands, it turns out that a part of mere lawful hand is examined.

Considering the problem that a hand with pieces easily exceeds the number of 100 moves, it turns out that players are bound to examine only a part of the legal moves.

It is assumed that human players cannot consider many matters in parallel. Moreover, a human does not count all legal moves and so it differs in this respect from a computer. In practice it turned out that the human search process is particularly

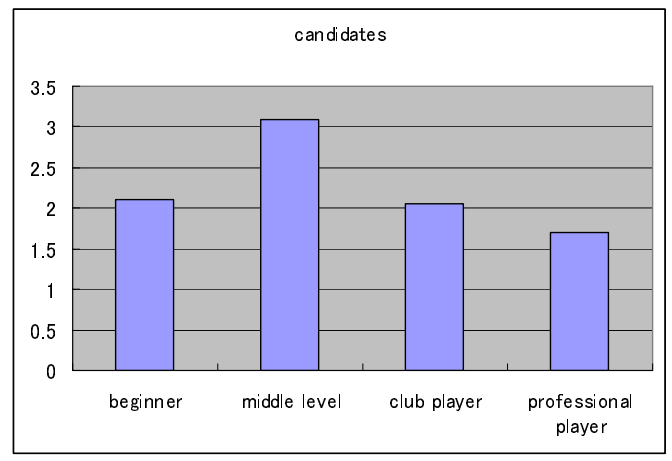

Fig. 1. Average number of the candidate move on next move test 
used for candidate moves when only a few moves exist. The move chosen is then evaluated by intuition.

\section{(2) Predict in linear}

Given the problem as shown in Figure 2. By the time it had to choose a next move the Shogi player under consideration was made to utter his decision-making process; the contents were recorded and analyzed [6].

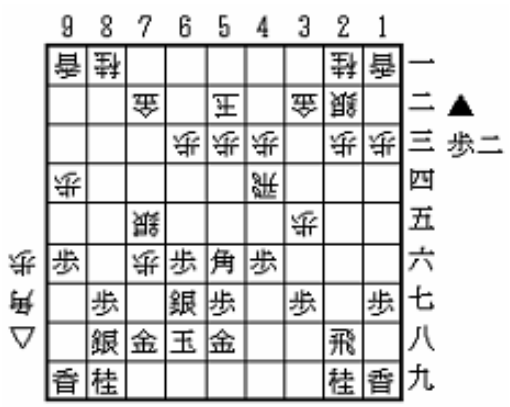

Fig. 2. A problem of next move test experiments

Figures 3-6 express by a search tree the move at which a player of a certain Shogi strength predicted the next move of Figure 2. Although there is a great difference in the contents of thought, it turned out that the form of a search tree has the almost same form. And, although there are some branches with candidate moves, the tree predicts almost linearly.

We believe that a move generation system for mimicking the thought processes of a human Shogi Player who advances and arrives at the point doing so linearly. In computer Shogi, the candidate moves are all the legal moves, and a game tree is constituted from a prediction by assuming all legal moves by Black and White. The

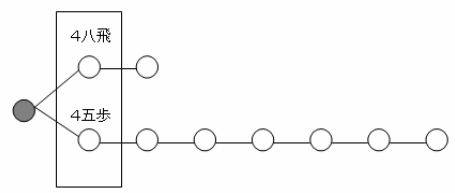

Fig. 3. A search tree of novice

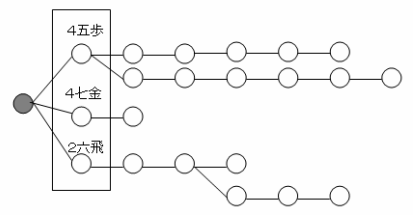

Fig. 5. A search tree of club player

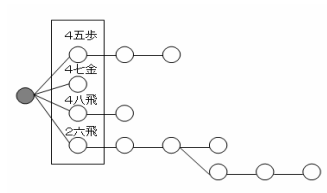

Fig. 4. A search tree of middle level player

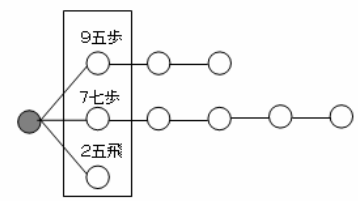

Fig. 6. A search tree of professional player 
program searches by the MIN-MAX method using a static evaluation function which assesses the advantage or disadvantage of the position. However, it is clear that a human does not do MIN-MAX calculation like a computer. A human player predicts almost linearly. He starts at the intuitively proper move, and predicts to confirm whether the future position becomes really good for himself.

\section{Selfish Search Based on Psychological Restrictions}

As seen in Section 2, a candidate move is extracted and then the search process peculiar to a human being is made. The process predicts linearly and is called "Selfish Search". So it is different from the search by a computer. We believe that this "selfish prediction" is a thought process that is peculiar to a human. It is bounded by psychological restrictions by the human being. The range of the target problem is narrow. If a problem is counted in all its possibilities, it is also possible that all the cases are performing a comprehensive search like a computer. For example, if it is a game with comparatively narrow search space such as TIC-TAC-TOE (e.g., by summarizing the symmetrical position or eliminating the move in which it loses simply), it will be possible to narrow the search space sharply. Analogously, a human will also count all the possibilities, and he will try to deduce a conclusion. However, the difficult games (the game of Go, Shogi, Chess, etc.) which have been played for a long time have a search space in which a human cannot search easily. In the large game tree of such a search space, a human gives up all searching, performs a "selfish search" within the limits which focus on some moves. He then can assume by "intuition", and is considered to determine a next move. Figure 7 expresses the number and speed of the prediction in relation to Shogi skill. The graph is from the result of an above-mentioned psychological experiment. According to this figure, it turns out that the speed of searching becomes quickly, so that the Shogi skill becomes high, and the number of searching is increasing according to it. However, the speed which a top professional player searches is about ten moves per minute. The space which can be searched within a limited time is considered to be about at most some hundred moves. Thus, it can be said that there is a psychological limit in a human's search speed.

Besides, a comparison of the search tree described by (2) in Section 2 shows that a human is performing as few linear predictions of a branch as possible. Furthermore, a human never calculates MIN-MAX-search for the all search trees with generating parallel branches from a certain node. The human advances by one reading at a time; many linear prediction results are compared simply, and a next move is determined so that it may become a straight line by a certain fixed view. In other words, a human can say that he is poor at performing a parallel prediction, and that he can perform only sequential predictions.

Such "a limit of searching speed" and "a limit of parallel search ability" are limits of functional calculation abilities of the human. I call this limit "psychological restrictions". If it thinks as mentioned above, it can be said that human thought is bound and prescribed by these psychological restrictions. "Selfish Search" which the human player is performing can be considered to be the optimized thought method in the "psychological restrictions" of the human. 


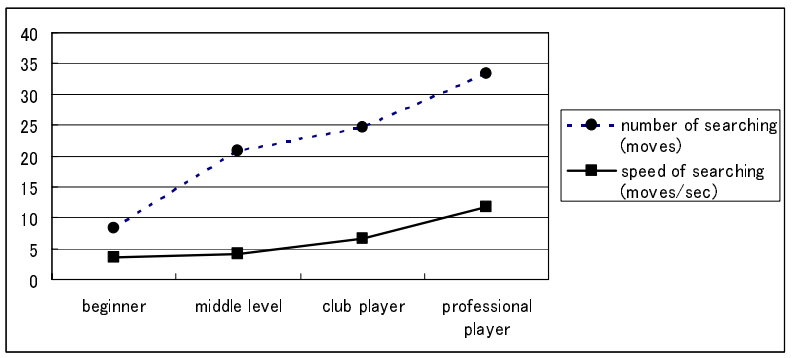

Fig. 7. Number and speed of searching for the strength on Shogi

\section{Proposal of Selfish Search System}

In advance of this selfish Search system, I have developed the knowledge driven Shogi system (HIT: Human Intuitive Thought) imitating human intuitive thought [10]. This system can generate a move by describing the intuitive knowledge which the expert of Shogi has in the form of a production rule, without searching. In HIT, a score is first given to all the legal moves by the production rule prepared beforehand. About 400 production rules are given to HIT now. By applying these rules, a score is given to all the legal moves Based on the given scores, the moves are sorted. The move which has the highest score is generated as a candidate move. A next move is determined by performing selfish prediction as shown in Figure 9 using the candidate move generated by HIT.

As shown in Figure 8, if a position is given, a score will be given to all the legal moves by HIT, and it will sort sequentially from a higher rank. It will become a candidate move if the higher lank of candidate move and within threshold $\alpha$. If the

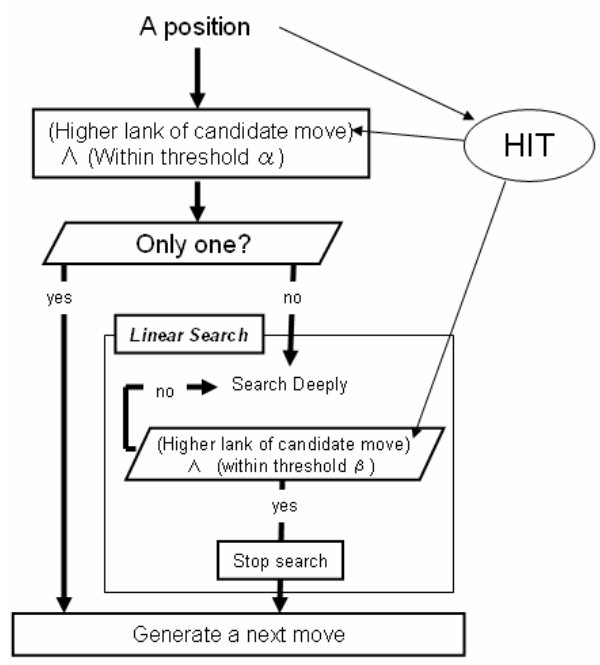

Fig. 8. Selfish Search Algorithm 
candidate is only one, the move is the next move. If there are two or more candidate moves, it will be searched deeply. This search is repeated until the difference between candidate moves reaches below the threshold value $\beta$. This repetition realizes linear search. This linear search is performed to all candidate moves, an evaluation function estimates the position of each termination, and the move of most a high score is chosen as a next move. Selfish search of human is imitated by following the series of procedure.

\section{Conclusion and Future Research}

This article investigated the main characteristics of human search. The concept of "selfish search" was introduced to describe the decision-making process of a human player. We explained that "selfish search" is based on psychological restrictions. Moreover, we considered a function of "selfish search" in the middle stage of a game and formulated on adequate learning process.

Future research will address a knowledge-driven type of a computer-aided design using "selfish search" in combination with a learning-support system for human players.

\section{References}

[1] Totsugeki-Tohoku: Science of the Mah-jongg, Kodansha (2004)

[2] de Groot, A.D.: Thought and Choice in Chess. Mouton Publishers, The Hague, The Netherlands (1965)

[3] Ito, T.: A Cognitive Processes on Playing Shogi. In: Game Programming Workshop in Japan '99, pp. 177-184 (1999)

[4] Ito, T., Matsubara, H., Grimbergen, R.: Cognitive Science Approach to Shogi Playing Processes (1) -Some Results on Memory Experiments. Information Processing Society of Japan Journal 43(10), 2998-3011 (2002)

[5] Ito, T., Matsubara, H., Grimbergen, R.: Cognitive Science Approach to Shogi Playing Processes (2) -Some Results on Next Move Test Experiments. Information Processing Society of Japan Journal 45(5), 1481-1492 (2004)

[6] Ito, T.: The Thought and Cognition on the Verbal Data of Shogi Experts, IPSJ SIG-GI12-2, pp.9-15 (2004)

[7] Ito, T.: Thinking of Computers vs Professional Players -Contemporary and Future of Computer Shogi. In: Game Programming Workshop in Japan 2005, pp. 40-47 (2005)

[8] Takizawa, T.: A Success of the Computer Shogi of the New Feeling by "Full Search" and Learning, and an Influence of the High-speed Algorithm. IPSJ Magazine 47(8), 875-881 (2006)

[9] Takizawa, T.: Contemporary Computer Shogi. IPSJ SIG-GI-16-1, pp.1-8 (May 2006)

[10] Ito, T.: A Shogi Program that Applies the Intuitive Thinking of Experts -HIT (Human Intuitive Thought) Shogi Project. Information Processing Society of Japan Journal 46(6), 1527-1532 (2005) 